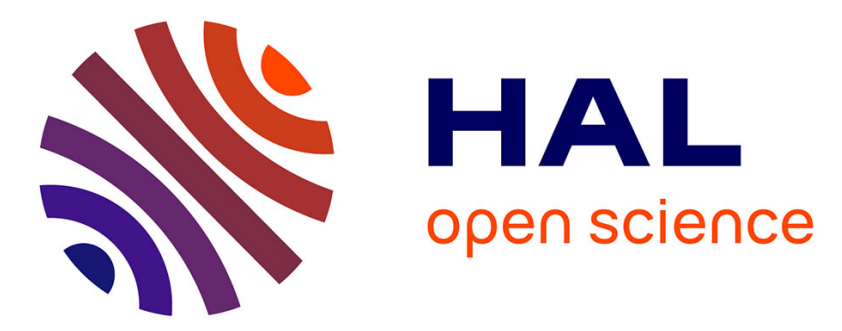

\title{
Implicit Lyapunov-Krasovski Functionals For Stability Analysis and Control Design of Time-Delay Systems
}

Andrey Polyakov, Denis Efimov, Wilfrid Perruquetti, Jean-Pierre Richard

\section{To cite this version:}

Andrey Polyakov, Denis Efimov, Wilfrid Perruquetti, Jean-Pierre Richard. Implicit LyapunovKrasovski Functionals For Stability Analysis and Control Design of Time-Delay Systems. IEEE Transactions on Automatic Control, 2015, pp.6. 10.1109/TAC.2015.2422451 . hal-01160061

\section{HAL Id: hal-01160061 \\ https://inria.hal.science/hal-01160061}

Submitted on 4 Jun 2015

HAL is a multi-disciplinary open access archive for the deposit and dissemination of scientific research documents, whether they are published or not. The documents may come from teaching and research institutions in France or abroad, or from public or private research centers.
L'archive ouverte pluridisciplinaire HAL, est destinée au dépôt et à la diffusion de documents scientifiques de niveau recherche, publiés ou non, émanant des établissements d'enseignement et de recherche français ou étrangers, des laboratoires publics ou privés. 


\title{
Implicit Lyapunov-Krasovski Functionals For Stability Analysis and Control Design of Time-Delay Systems
}

\author{
A. Polyakov, D. Efimov, W. Perruquetti, J.-P. Richard
}

\begin{abstract}
The method of Implicit Lyapunov-Krasovski Functional (ILKF) for stability analysis of time-delay systems is introduced. Theorems on Lyapunov, asymptotic, (hyper) exponential, finite-time and fixed-time stability analysis using ILKF are presented. The hyper exponential stabilization algorithm for a time-delay system is presented. The theoretical result is supported by numerical simulation.
\end{abstract}

\section{INTRODUCTION}

The celebrated Second Lyapunov Method was originally founded on the so-called energetic approach to stability analysis. It considers any positive definite function as possible energetic characteristic ("energy") of a dynamic system and the evolution of this "energy" in time is studied. If a dynamic system has an energetic function, which is decreasing (or strictly decreasing) along any trajectory of the system, then this system has some stability property and the corresponding energetic function is called a Lyapunov function.

The Lyapunov function method is one of the main theoretical tools for stability analysis and control synthesis of nonlinear systems ([2], [21]). This method allows the stability properties of the system to be studied without finding its solutions. The extension of the Lyapunov's ideas to the case of time-delay systems is given by Lyapunov-Razumikhin and Lyapunov-Krasovski theorems, see, for example, [15], [10], [25], [9], [17]. The corresponding energetic function becomes a functional in the last case. Finding the appropriate Lyapunov (-Krasovski) functions is a difficult problem in general case.

In addition to qualitative analysis of stability, control theory is also interested in quantitative analysis of the convergence rate. In linear case the concept of exponential stability can be utilized for this purpose. Nonlinear control systems may demonstrate faster (e.g. hyper exponential) convergence. In particular, nonlinear systems can be finite-time [26] and fixedtime stable [22]. Finite-time control problem is a subject of intensive research in the last years; e.g., see [11], [3], [13], [19], [21]. The method of Lyapunov(-Krasovski) functions helps to estimate the rate of convergence and to adjust the control parameters in order to provide the desired rate.

Andrey Polyakov, Denis Efimov, Wilfrid Perruquetti and J.-P. Richard are with Non-A INRIA - LNE, Parc Scientifique de la Haute Borne 40, avenue Halley Bat.A, Park Plaza 59650 Villeneuve d'Ascq, France (e-mail: andrey.polyakov@inria.fr, denis.efimov@inria.fr, wilfrid.perruqetti@inria.fr, jean-pierre.richard@ec-lille.fr). They are also with LAGIS UMR CNRS 8219, Ecole Centrale de Lille, BP 48, Cité Scientifique, 59651 Villeneuve-d'Ascq, France.
Recently [23], the Implicit Lyapunov Function (ILF) method has been developed for non-asymptotic (finite-time and fixedtime) analysis. This method allows the control to be designed together with a Lyapunov function of the closed-loop system. A simple procedure for parameter tuning based on Linear Matrix Inequalities (LMIs) was developed for linear plants.

The ideas, which underline the ILF method, were initially introduced for control synthesis problems in [16]. The corresponding design methodology was called controllability function method. In order to be more precise we follow more recent terminology of [1]. The ILF method uses Lyapunov function defined in the implicit form by some algebraic equations. Stability analysis in this case does not require solution of this equation, since the Implicit function theorem (see, for example, [6]) helps to check all stability conditions by analyzing the algebraic equation directly.

This technical note extends the concept of ILF to the case of time-delay systems. The stability theorems presented in the paper introduce the ILKF method for Lyapunov, asymptotic, (hyper) exponential and non-asymptotic (finite-time and fixedtime) stability analysis of time-delay systems. They allow us to define the Lyapunov-Krasovski functionals in the implicit form. The developed method is demonstrated on the control design problem for hyper exponential stabilization of a special time-delay linear system. The procedure to design of control parameters is presented in the LMI form.

\section{NOTATION}

Through the paper the following notation will be used:

- $\mathbb{R}$ is the field of real numbers, $\mathbb{R}_{+}=\{x \in \mathbb{R}: x>0\}$;

- $\|\cdot\|$ is the Euclidian norm in $\mathbb{R}^{n}$;

- $\operatorname{diag}\left\{\lambda_{i}\right\}_{i=1}^{n}$ is the diagonal matrix with the elements $\lambda_{i}$ on the main diagonal;

- a positive definite continuous function $\sigma: \mathbb{R} \rightarrow \mathbb{R}$ belongs to the class $\mathcal{K}$ if it is strictly increasing on $\mathbb{R}_{+}$ and $\sigma(0)=0$; if additionally it is radially unbounded then $\sigma$ belongs to $\mathcal{K}_{\infty}$;

- $\mathbb{C}_{h}$ is the space of continuous functions $[-h, 0] \rightarrow$ $\mathbb{R}^{n}$ with the norm $\|\cdot\|_{h}$ defined as follows $\|\varphi\|_{h}=$ $\max _{t \in[-h, 0]}\|\varphi(t)\|$ for $\varphi \in \mathbb{C}_{h}$;

- $\mathbb{C}_{h}^{0}:=\left\{\varphi \in \mathbb{C}_{h}: \varphi(0)=0\right\}$ is a subspace of $\mathbb{C}_{h}$.

- for $P>0$ the matrix $P^{1 / 2}:=B$ is such that $B^{2}=P$ and $P^{-1 / 2}=\left[P^{1 / 2}\right]^{-1}$;

- $\lambda_{\min }(P)$ and $\lambda_{\max }(P)$ are minimal and maximal eigenvalues of symmetric matrix $P \in \mathbb{R}^{n \times n}$, respectively. 
- if $P \in \mathbb{R}^{n \times n}$ is symmetric then the inequalities $P>0$ $(P<0)$ and $P \geq 0(P \leq 0)$ means that $P$ is positive (negative) definite and semidefinite, respectively;

\section{Stability Definitions}

Consider the system of the form

$$
\begin{aligned}
& \dot{x}(t)=f\left(t, x_{h}(t)\right), \quad t \in \mathbb{R}_{+} \\
& x(t)=\varphi(t) \in \mathbb{C}_{h}, \quad t \in[-h, 0]
\end{aligned}
$$

where $x \in \mathbb{R}^{n}, x_{h}(t) \in \mathbb{C}_{h}$ is the state function defined by means of the formula $x_{h}(t):=x(t+\tau)$ with $\tau \in[-h, 0]$ with $h \in \mathbb{R}_{+}$(time delay) and $f: \mathbb{R}_{+} \times \mathbb{C}_{h} \rightarrow \mathbb{R}^{n}$ is a continuous operator. Note that the continuity of $f$ implies, at least, local existence of solutions of the system (1) due to Theorem 2.1, page 41 from [12]. The advanced detailed analysis of the existence or uniqueness properties of the solutions goes out of the scope of this paper. Assume that the origin is an equilibrium point of the system (1), i.e. $f(t, 0)=0$ for all $t \in \mathbb{R}_{+}$. A solution of the system (4) with the initial function $\varphi \in \mathbb{C}_{h}$ is denoted by $x(t, \varphi)$.

The definitions of Lyapunov, asymptotic and exponential stability are well-known, we refer the reader, for example, to [12]. Interested in quantitative analysis of stability characterized by rate of convergence, we briefly discuss some related notions. Note that this paper deals only with strong uniform stability properties of the system (1). The words "strong uniform" are omitted below for simplicity of presentation.

Let us introduce the functions $\rho_{r}: \mathbb{R} \rightarrow \mathbb{R}$ by the following recursive formula

$$
\rho_{0}(z)=z, \quad \rho_{r}(z)=e^{\rho_{r-1}(z)},
$$

where $r \geq 1$ is integer number. The next definition presents hyper exponential asymptotic stability [24]. It follows the ideas of hyperpower (see, [18]).

Definition 1: The origin of the system (1) is said to be hyper exponentially stable of degree $r \geq 1$, if it is asymptotically stable, $\exists \sigma \in \mathcal{K}_{\infty}$ and $\exists \alpha \in \mathbb{R}_{+}$such that the inequality $\|x(t, \varphi)\| \leq \sigma\left(\|\varphi\|_{h}\right) e^{-\rho_{r}(\alpha t)}, t \in \mathbb{R}_{+}$holds for all $\varphi \in \mathbb{C}_{h}:\|\varphi\|_{h}<\delta$. If $\delta=+\infty$ then the origin of the system (1) is said to be globally hyper exponentially stable.

For $r=0$ the hyper exponential stability becomes exponential one [12]. The number $\alpha$ is called the decay rate of hyper exponential convergence. For example, the following delayfree system $\dot{x}(t)=-(1-\ln (|x(t)|)) x(t), x \in \mathbb{R}$ is locally hyper exponentially stable with the rate $\alpha=1$ and degree $r=1$, i.e. $\|x(t)\| \leq \sigma\left(\left|x_{0}\right|\right) e^{-\rho_{1}(t)}$, where $\rho_{1}(t)=e^{t}$.

The next two definitions present a stability with nonasymptotic reaching phase.

Definition 2 ([19]): The origin of the system (1) is said to be finite-time stable, if it is Lyapunov stable and for any $\varphi \in \mathbb{C}_{h}:\|\varphi\|_{h}<\delta$ there exists $0 \leq T_{0}(\varphi)<+\infty$, such that $\|x(t, \varphi)\|=0$ for $t \geq T_{0}(\varphi)$. If $\delta=+\infty$ then the origin of the system (1) is said to be globally finitetime stable. The functional $T: \mathbb{C}_{[-h, 0]} \rightarrow \mathbb{R}_{+}$defined as $T(\varphi)=\inf \left\{T_{0}(\varphi) \geq 0: x(t, \varphi)=0, \forall t>T(\varphi)\right\}$ is called the settling-time functional [19] of the system (1).

Examples of finite-time stable systems of the form (1) are given in [19]. Note that the Lyapunov-Razumikhin approach to finite-time stability analysis is inconsistent in the general case [8].

The next definition extends the concept of fixed-time stability [22] to time-delay systems.

Definition 3: The origin of the system (1) is said to be fixed-time stable, if it is finite-time stable and the settlingtime functional $T: \mathbb{C}_{h} \rightarrow \mathbb{R}_{+}$is bounded, i.e. there exists $T_{\max } \in \mathbb{R}_{+}$such that $\sup T(\varphi)=T_{\max }$ for some $\varphi \in \mathbb{C}_{h}:\|\varphi\|_{h}<\delta$

$\delta \in \mathbb{R}_{+}$. If $\delta=+\infty$ then the origin of the system (1) is said to be globally fixed-time stable.

The right-hand sides of finite-time and fixed-time stable systems are non-Lipschitz continuous [20] or even discontinuous [22]. Such models appear for mechanical systems with dry friction (see, for example, [4]). The phyper exponentially stable system presented above is also non-Lipschitz at the origin.

\section{Stability Analysis of Time-Delay Systems USING IMPLICIT LYAPUNOV-KRASOVSKI FUNCTIONAL}

\section{A. Implicit functions and operators}

Below we utilize a special class of functions introduced by the following definition.

Definition 4: The function $q: \mathbb{R}_{+}^{2} \rightarrow \mathbb{R},(\sigma, s) \rightarrow q(\sigma, s)$ is said to be of the class $\mathcal{I} \mathcal{K}_{\infty}$ iff 1) $q$ is continuous on $\mathbb{R}_{+}^{2}$;2) for any $s \in \mathbb{R}_{+}$there exists $\sigma \in \mathbb{R}_{+}$such that $q(\sigma, s)=0$; 3 ) for any fixed $s \in \mathbb{R}_{+}$the function $q(\cdot, s)$ is strictly decreasing on $\left.\mathbb{R}_{+} ; 4\right)$ for any fixed $\sigma \in \mathbb{R}_{+}$the function $q(\sigma, \cdot)$ is strictly increasing on $\mathbb{R}_{+}$; 5) $\lim \sigma=0, \lim s=0, \lim _{s \rightarrow+\infty} \sigma=+\infty$,

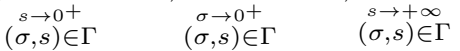

where $\Gamma=\left\{(\sigma, s) \in \mathbb{R}_{+}^{2}: q(\sigma, s)=0\right\}$.

If $q \in \mathcal{I} \mathcal{K}_{\infty}$ then there exists a unique function $\sigma \in \mathcal{K}_{\infty}$, such that $q(\sigma(s), s)=0$ for all $s \in \mathbb{R}_{+}$. Indeed, the condition 2 ) claims that there exists a function $\sigma: \mathbb{R}_{+} \rightarrow \mathbb{R}_{+}$such that $q(\sigma(s), s)=0$ for any $s \in \mathbb{R}_{+}$. The condition 3 ) implies the uniqueness of this function. The implicit function theorem [14] and the conditions 1) - 4) guarantee continuity of $\sigma$ in $\mathbb{R}_{+}$. In order to show that $\sigma$ is strictly increasing let us take two arbitrary numbers $s_{1}, s_{2} \in \mathbb{R}_{+}$such that $s_{1}<s_{2}$ and denote $\sigma_{1}=\sigma\left(s_{1}\right)$ and $\sigma_{2}=\sigma\left(s_{2}\right)$. The condition 4) implies $q\left(\sigma_{1}, s_{1}\right)=0<q\left(\sigma_{1}, s_{2}\right)$. Hence, $q\left(\sigma_{2}, s_{2}\right)=0<q\left(\sigma_{1}, s_{2}\right)$ and due to the condition 3) we have $\sigma_{1}<\sigma_{2}$. Finally, the condition 5) guarantees that $\sigma(s) \rightarrow+\infty$ as $s \rightarrow+\infty$ and the function $\sigma$ can be prolonged by continuity to the origin as follows $\sigma(0)=0$

Recall that the operator $g: \mathbb{Z} \rightarrow \mathbb{Y}$, where $\mathbb{Z}$ and $\mathbb{Y}$ are Banach spaces, is called F-differentiable (Frèchet differentiable) at $z_{0} \in \mathbb{Z}$ if there exists a linear bounded operator $D g_{z_{0}}: \mathbb{Z} \rightarrow \mathbb{Y}$

$$
\frac{\left\|g(z)-g\left(z_{0}\right)-D g_{z_{0}}\left(z-z_{0}\right)\right\|_{\mathbb{Y}}}{\left\|z-z_{0}\right\|_{\mathbb{Z}}} \rightarrow 0 \quad \text { as } \quad\left\|z-z_{0}\right\|_{\mathbb{Z}} \rightarrow 0
$$

where $\|\cdot\|_{\mathbb{Z}}$ and $\|\cdot\|_{\mathbb{Y}}$ are norms in the Banach spaces $\mathbb{Z}$ and $\mathbb{Y}$, respectively.

Theorem 1 ([7]): Let $\mathbb{Z}, \mathbb{Y}$ and $\mathbb{Q}$ be Banach spaces. Let $g$ : $\mathbb{Z} \times \mathbb{Y} \rightarrow \mathbb{Q}$ be a continuous operator such that 1) $g\left(z_{0}, y_{0}\right)=0$ for some $z_{0} \in \mathbb{Z}$ and $y_{0} \in \mathbb{Y}$; 2) $g$ is F-differentiable in the neighborhood of the point $\left(z_{0}, y_{0}\right)$; 3) $D g_{z_{0}}^{\prime}$ is invertible, 
where $g^{\prime}(z):=g\left(z, y_{0}\right)$. Then there exists a unique operator $f: \mathbb{Y} \rightarrow \mathbb{Z}$ defined in an open neighborhood $U_{0}$ of $y_{0}$ such that $g(f(y), y)=0$ for all $y \in U_{0}$. Moreover, if $g$ is continuously F-differentiable at a neighborhood of $\left(z_{0}, y_{0}\right)$ then $f$ is also continuously differentiable at some neighborhood of $y_{0}$.

\section{B. Theorems on Implicit Lyapunov-Krasovski Functional}

The next theorem provides a background for stability analysis of time-delay systems using ILKF.

Theorem 2: If there exists a continuous functional $Q: \mathbb{R}_{+} \times$ $\mathbb{C}_{h} \rightarrow \mathbb{R}$ such that:

C1) $Q$ is continuously F-differentiable on $\mathbb{R}_{+} \times \mathbb{C}_{h}$;

$C 2)$ for any $y \in \mathbb{C}_{h}$ there exists $V \in \mathbb{R}_{+}$such that $Q(V, y)=$ 0 ;

C3) $\frac{\partial Q(V, y)}{\partial V}<0$ for all $V \in \mathbb{R}_{+}$and $y \in \mathbb{C}_{h}$;

C4) there exist $q_{i} \in \mathcal{I} \mathcal{K}_{\infty}, i=1,2$ such that for all $V \in \mathbb{R}_{+}$

$$
\begin{aligned}
q_{1}(V,\|y(0)\|) \leq Q(V, y), & \forall y \in \mathbb{C}_{h} \backslash \mathbb{C}_{h}^{0}, \\
Q(V, y) \leq q_{2}\left(V,\|y\|_{h}\right), & \forall y \in \mathbb{C}_{h} \backslash\{0\},
\end{aligned}
$$

C5) for all $\left(V, x_{h}(t)\right) \in \Omega$ such that $x(t)$ satisfies (1) we have

$$
\frac{\partial Q\left(V, x_{h}(t)\right)}{\partial t} \leq-\sigma(\|x(t)\|), \quad \forall t \in \mathbb{R}_{+},
$$

where $\sigma: \mathbb{R} \rightarrow \mathbb{R}$ and

$$
\Omega=\left\{(V, y) \in \mathbb{R}_{+} \times \mathbb{C}_{h}: Q(V, y)=0\right\} .
$$

Then the origin of the system (1) is Lyapunov stable if $\sigma$ is a nonnegative function and asymptotically stable if $\sigma \in \mathcal{K}$.

Proof: In order to prove the theorem let us show that there exists a functional $V: \mathbb{C}_{h} \rightarrow \mathbb{R}_{+} \cup\{0\}$ that satisfies conditions of classical stability theorem for functional-differential equations (for example, Theorem 2.1 on page 105 from [12]).

I. Given $\tilde{y} \in \mathbb{C}_{h}$ let us consider the scalar function $Q(\cdot, \tilde{y})$ and show that it has exactly one zero on $\mathbb{R}_{+}$. The condition $C 2$ ) claims that there exists at least one $\tilde{V} \in \mathbb{R}_{+}$such that $Q(\tilde{V}, \tilde{y})=0$. The conditions $C 1)$ and C3) imply that the function $Q(\cdot, \tilde{y})$ is monotone, i.e. $\tilde{V}$ is the unique zero in $\mathbb{R}_{+}$. Therefore, there exists a unique functional $V: \mathbb{C}_{h} \backslash\{0\} \rightarrow$ $\mathbb{R}_{+}$such that $Q(V(y), y)=0$ for any $y \in \mathbb{C}_{h}$. Theorem 1 guarantee that $V$ is continuously F-differentiable functional on $\mathbb{C}_{h} \backslash\{0\}$.

II. Since $q_{1}, q_{2} \in \mathcal{I} \mathcal{K}_{\infty}$ then there exists $\sigma_{1}, \sigma_{2} \in \mathcal{K}_{\infty}$ such that $q_{1}\left(\sigma_{1}(s), s\right)=0$ and $q_{2}\left(\sigma_{2}(s), s\right)=0$ for all $s \in$ $\mathbb{R}_{+}$. Note that the functions $\sigma_{1}$ and $\sigma_{2}$ can be extended by continuity to $\mathbb{R}_{+} \cup\{0\}$ as follows $\sigma_{1}(0)=0$ and $\sigma_{2}(0)=0$.

The condition C4) implies $q_{1}(V(y),\|y(0)\|) \leq$ $Q(V(y), y)=0=q_{1}\left(\sigma_{1}(\|y(0)\|),\|y(0)\|\right)$ for all $y \in \mathbb{C}_{h} \backslash \mathbb{C}_{h}^{0}$ and $q_{2}\left(\sigma_{2}\left(\|y\|_{h}\right),\|y\|_{h}\right)=0=Q(V(y), y) \leq$ $q_{2}\left(V(y),\|y\|_{h}\right)$ for all $y \in \mathbb{C}_{h} \backslash\{0\}$. Due to the condition 3) of Definition 4 the obtained inequalities guarantee $\sigma_{1}(\|y(0)\|) \leq V(y)$ for all $y \in \mathbb{C}_{h} \backslash \mathbb{C}_{h}^{0}$ and $V(y) \leq \sigma_{2}\left(\|y\|_{h}\right)$ for all $y \in \mathbb{C}_{h} \backslash\{0\}$. Hence, the functional $V$ can be extended by continuity to $\mathbb{C}_{h}$ as follows $V(0)=0$. Taking into account $V(y) \in \mathbb{R}_{+}$for any $y \in \mathbb{C}_{h}^{0} \backslash\{0\}$ we derive $\sigma_{1}(\|y(0)\|) \leq V(y) \leq \sigma_{2}\left(\|y\|_{h}\right)$ for all $y \in \mathbb{C}_{h}$.

III. Let $x(t)$ be a solution of (1) and $x_{h}(t) \in \mathbb{C}_{h}$ be defined by $x_{h}(t):=x(t+\tau)$ with $\tau \in[-h, 0]$. Let us consider the functions $\tilde{V}(t):=V\left(x_{h}(t)\right)$ and $\tilde{Q}(V, t):=Q\left(V, x_{h}(t)\right)$ for $t \in \mathbb{R}_{+}$. Obviously, $\tilde{Q}(\tilde{V}(t), t)=0$ for all $t \in \mathbb{R}_{+}$such that $x_{h}(t) \neq 0$, and the classical implicit function theorem [6] for Euclidean spaces implies $\frac{d}{d t} \tilde{V}(t)=-\left[\frac{\partial \tilde{Q}}{\partial V}\right]^{-1} \frac{\partial \tilde{Q}}{\partial t}$. Taking into account continuity of the functional $V$ at $0 \in \mathbb{C}_{h}$ and the condition $C 5$ ) we derive

$$
\dot{V}\left(x_{h}(t)\right)=-\left[\frac{\partial Q\left(V, x_{h}(t)\right)}{\partial V}\right]^{-1} \frac{\partial Q\left(V, x_{h}(t)\right)}{\partial t} \leq-\tilde{\sigma}(\|x(t)\|),
$$

where $\tilde{\sigma}=\left[-\frac{\partial Q}{\partial V}\right]^{-1} \sigma$ and $t \in \mathbb{R}_{+}$. Finally, applying Theorem 2.1, page 105 from [12] we finish the proof.

Theorem 2 introduces an additional flexibility to the design of Lyapunov-Krasovski functionals provided the possibility of their implicit definition. However, the implicit design requires a more complicated analysis based on conditions C1)-C5).

Note that the condition C3) can be relaxed as follows $\frac{\partial Q(V, y)}{\partial V}<0$ for all $(V, y) \in \Omega$. It is worth stressing out that, similarly to the classical method of Lyapunov-Krasovski functionals, the condition C5) can be checked using the righthand side of the equation (1) only (see the next section).

Theorem 3: If there exists a continuous functional $Q$ : $\mathbb{R}_{+} \times \mathbb{C}_{h} \rightarrow \mathbb{R}$, which satisfies the conditions C1)-C4) of Theorem 2 and the condition:

C5bis) for all $\left(V, x_{h}(t)\right) \in \Omega$ such that $0<V<V^{\max }$ and $x_{h}(t)$ satisfies (1) we have

$$
\frac{\partial Q\left(V, x_{h}(t)\right)}{\partial t} \leq \alpha V^{1-\mu} \frac{\partial Q\left(V, x_{h}(t)\right)}{\partial V}, \quad t \in \mathbb{R}_{+},
$$

where $\alpha, V^{\max } \in \mathbb{R}_{+}, \mu \in(0,1]$ and $\Omega$ is defined by (2).

Then the origin of system (1) is finite-time stable (globally finite-time stable if $V^{\max }=+\infty$ ) with the following settling time estimate:

$$
T(\varphi) \leq \frac{V_{0}^{\mu}}{\alpha \mu}, \quad V_{0} \in \mathbb{R}_{+}: Q\left(V_{0}, \varphi\right)=0 .
$$

Proof: Repeating the proof of Theorem 2 we show existence of a proper Lyapunov-Krasovski functional $V$ : $\mathbb{C}_{h} \rightarrow \mathbb{R}_{+} \cup\{0\}$ implicitly defined by the equation $Q(V, y)=$ 0 . The condition C5bis) and the implicit function theorem [6] provide $\dot{V}\left(x_{h}(t)\right)=-\left[\frac{\partial Q\left(V, x_{h}(t)\right)}{\partial V}\right]^{-1} \frac{\partial Q\left(V, x_{h}(t)\right)}{\partial t} \leq$ $\left.-\alpha V^{1-\mu}\left(x_{h}(t)\right)\right)$. Hence, Proposition 4 presented in [19] implies finite-time stability of the origin of the system (1) with the settling time estimate (3).

Theorem 4: Let there exist two continuous functionals $Q_{1}$ : $\mathbb{R}_{+} \times \mathbb{C}_{h} \rightarrow \mathbb{R}$ and $Q_{2}: \mathbb{R}_{+} \times \mathbb{C}_{h} \rightarrow \mathbb{R}$, which satisfy the conditions (1)-C4) of Theorem 2. If the functional $Q_{1}$ satisfies the condition C5bis) of Theorem 3 with $\sigma(V)=\alpha V^{1-\mu}, \alpha \in$ $\mathbb{R}_{+}, \mu \in(0,1], V^{\max }=1$, and

C6) $Q_{1}(1, y)=Q_{2}(1, y)$ for all $y \in \mathbb{C}_{h}:\|y\|<\delta$, where $\delta \in \mathbb{R}_{+}$is some constant;

C7) for all $\left(V, x_{h}(t)\right) \in \Omega_{2}$ such that $V \geq 1$ and $x_{h}(t)$ satisfies (1) we have

$$
\frac{\partial Q_{2}\left(V, x_{h}(t)\right)}{\partial t} \leq \beta V^{1+\nu} \frac{\partial Q_{2}\left(V, x_{h}(t)\right)}{\partial V}, \quad t \in \mathbb{R}_{+}
$$

where $\beta, \nu \in \mathbb{R}_{+}$and $\Omega_{2}$ is defined by (2) with $Q=Q_{2}$, then the origin of system (1) is (globally) fixed-time stable (if $\delta=+\infty)$ with the settling time estimate: $T(\varphi) \leq \frac{1}{\alpha \mu}+\frac{1}{\beta \nu}$.

Proof: The conditions (1)-C4) of Theorem 2 guarantees existence of proper Lyapunov-Krasovski functionals $V_{1}$ : 
$\mathbb{C}_{h} \rightarrow \mathbb{R}_{+} \cup\{0\}$ and $V_{2}: \mathbb{C}_{h} \rightarrow \mathbb{R}_{+} \cup\{0\}$ implicitly defined by the equations $Q_{1}\left(V_{1}, y\right)=0$ and $Q_{2}\left(V_{2}, y\right)=0$ for any $y \in \mathbb{C}_{h}$. Given $y_{0} \in \mathbb{C}_{h}$ the inequality $V_{1}\left(y_{0}\right) \geq 1$ holds if and only if $V_{2}\left(y_{0}\right) \geq 1$. Indeed, if $V_{1}\left(y_{0}\right) \geq 1$ then the conditions C3) and C6) imply $Q_{2}\left(1, y_{0}\right)=Q_{1}\left(1, y_{0}\right) \geq$ $Q_{1}\left(V_{1}\left(y_{0}\right), y_{0}\right)=0=Q_{2}\left(V_{2}\left(y_{0}\right), y_{0}\right)$ and $V_{2}\left(y_{0}\right) \geq 1$. Analogously, given $y_{0} \in \mathbb{C}_{h}$ the inequality $V_{1}\left(y_{0}\right)<1$ holds if and only if $V_{2}\left(y_{0}\right)<1$.

The condition C7) implies $\dot{V}_{2}\left(x_{h}(t)\right) \leq-\beta V_{2}^{1+\nu}\left(x_{h}(t)\right)$ for $V_{2} \geq 1$. Hence, for $V_{2}\left(x_{h}(0)\right)>1$ we have $V_{2}\left(x_{h}(t)\right)<1$, $\forall t>T_{2}=\frac{1}{\beta \nu}$.

The condition C5bis) of Theorem 3 implies that if $V_{1}\left(x_{h}\left(t_{1}\right)\right)<1$ for some $t_{1} \in \mathbb{R}_{+} \cup\{0\}$, then we have $V_{1}\left(x_{h}(t)\right)=0, \forall t>t_{1}+T_{1}$, where $T_{1}=\frac{1}{\alpha \mu}$.

Implicit Lyapunov-Krasovski functional-based analysis of exponential and hyper exponential stability requires some additional restrictions to the class of functionals to be used.

Theorem 5: If there exist a continuous functional $Q: \mathbb{R}_{+} \times$ $\mathbb{C}_{h} \rightarrow \mathbb{R}$, which satisfies the conditions C1) - C4) of Theorem 2 and the conditions:

$\left.C 4^{*}\right)$ there exists $c \in \mathbb{R}_{+}$such that $q_{1}(c s, s) \geq 0$ for $0<c s<$ $V_{\max }$, where $q_{1}$ is defined by the condition $\left.C 4\right)$ of Theorem 2 and $V^{\max } \in \mathbb{R}_{+}$is some constant number;

$\left.C 5^{*}\right)$ for all $\left(V, x_{h}(t)\right) \in \Omega$ and $0<V<V^{\max }$ such that $x(t)$ satisfies (1) we have

$$
\frac{\partial Q\left(V, x_{h}(t)\right)}{\partial t} \leq \alpha p(V) V \frac{\partial Q\left(V, x_{h}(t)\right)}{\partial V}, \quad \forall t \in \mathbb{R}_{+},
$$

where $\alpha \in \mathbb{R}_{+}$is some constant number, then the origin of system (1) is

- exponentially stable if $p(V)=1$;

- hyper exponentially stable of degree $r=1$ if

$$
p(V)=\left\{\begin{array}{lll}
1+\ln (V) & \text { for } & V>1 \\
1-\ln (V) & \text { for } & V \leq 1
\end{array}\right.
$$

with the convergence rate $\alpha$. The global (hyper) exponential stability is provided if $V^{\max }=+\infty$.

Proof: Repeating the proof of Theorem 2 we show existence of a proper Lyapunov-Krasovski functional $V: \mathbb{C}_{h} \rightarrow$ $\mathbb{R}_{+} \cup\{0\}$ implicitly defined by the equation $Q(V, y)=0$. The condition $C 4^{*}$ implies that $q_{1}(c s, s) \geq q_{1}\left(\sigma_{1}, s\right)$ for all $\left(\sigma_{1}, s\right) \in \mathbb{R}_{+}^{2}: q_{1}\left(\sigma_{1}, s\right)=0$. Hence, due to the condition 3) of Definition 4 a function $\sigma_{1} \in \mathcal{K}_{\infty}$ such that $q_{1}\left(\sigma_{1}(s), s\right)=0, s \in \mathbb{R}_{+}$satisfies the inequality $c s \leq \sigma_{1}(s)$ for all $s \in \mathbb{R}_{+}: \sigma_{1}(s) \leq V_{\max }$.

Let us denote for shortness $V(t)=V\left(x_{h}(t)\right)$ and $V_{0}=V\left(x_{h}(0)\right)$. The condition $C 5^{*}$ ) implies $\dot{V}(t) \leq$ $-\alpha p(V(t)) V(t)$ along the trajectories of (1). For $p(V)=1$ we have $V(t) \leq V_{0} e^{-\alpha t}$. For $p(V)=1+\ln (V)$ and $V_{0}>1$ we derive $V(t) \leq e^{e^{-\alpha t} \ln \left(e V_{0}\right)-1} \leq V_{0} e^{1-e^{\alpha t}}, \quad 0 \leq$ $t \leq T_{1}$, where $T_{1}:=\alpha^{-1} \ln \left(\ln \left(e V_{0}\right)\right)$ and $V\left(T_{1}\right) \leq 1$. For $p(V)=1-\ln (V)$ and $V_{0} \leq 1$ we obtain $V(t) \leq$ $e^{e^{\alpha t} \ln \left(V_{0} / e\right)+1} \leq V_{0} e^{1-e^{\alpha t}}, \forall t>0$. Taking into account $c\|y(0)\| \leq \sigma_{1}(\|y(0)\|) \leq V(y)$ we finish the proof of the (hyper) exponential stability.

The next section presents an example of control design using Theorem 5 .

\section{EXAmPle: HyPER EXPONENTIAL STABILIZATION}

\section{A. Problem formulation}

Let us consider a single input control system of the form

$$
\left\{\begin{array}{l}
\dot{x}(t)=A_{0} x(t)+A_{1} x(t-h)+b u(t), \quad t>0, \\
x(t)=\varphi(t) \in \mathbb{C}_{h}, \quad t \in[-h, 0],
\end{array}\right.
$$

where $x \in \mathbb{R}^{n}$ is the state vector, $u \in \mathbb{R}$ is a control input, $A_{0}, A_{1} \in \mathbb{R}^{n \times n}$ are the system matrices of the form $A_{0}=$ $\left(\begin{array}{cc}0 & I_{n-1} \\ 0 & 0\end{array}\right), A_{1}=\operatorname{diag}\left\{a_{i}\right\}_{i=1}^{n}, a_{i} \in \mathbb{R}, i=1,2, \ldots, n$ and the vector $b \in \mathbb{R}^{n \times 1}$ is of the form $b=(0,0, \ldots, 1)^{\top}$.

The problem is to stabilize hyper exponentially the origin of the system (4).

\section{B. Hyper Exponential Control Design}

Introduce the Implicit Lyapunov-Krasovski Functional (ILKF) by the equality

$$
\begin{gathered}
Q(V, y):=-1+y^{\top}(0) D\left(\frac{1}{V}\right) P D\left(\frac{1}{V}\right) y(0)+ \\
\int_{-h}^{0}\left(\frac{e}{V}\right)^{\mu \frac{2 \tau+h}{h}} y^{\top}(\tau) D\left(\frac{1}{V}\right) S D\left(\frac{1}{V}\right) y(\tau) d \tau,
\end{gathered}
$$

where $\mu \in(0,1)$, the diagonal matrix $D(\cdot)$ is defined by $D(\lambda)=\operatorname{diag}\left\{\lambda^{1+\mu(n-i)}\right\}_{i=1}^{n}$ for $\lambda \in \mathbb{R}_{+}$and $0<P=$ $P^{\top} \in \mathbb{R}^{n \times n}, 0<S=S^{\top} \in \mathbb{R}^{n \times n}$. Denote $H:=$ $\operatorname{diag}\{1+\mu(n-i)\}_{i=1}^{n}$.

If $\mu=0$ then the equation $Q(V, y)=0$ provides the well-known Lyapunov-Krasovski functional: $V(y)=$ $\sqrt{y^{\top}(0) P y(0)+\int_{-h}^{0} y^{\top}(\tau) S y(\tau) d \tau}$.

The next proposition presents an algorithm of the ILKFbased control design.

Proposition 1: If $X \in \mathbb{R}^{n \times n}, Z \in \mathbb{R}^{n \times n}$ and $y \in \mathbb{R}^{1 \times n}$ satisfy the following system of LMIs:

$$
\left(\begin{array}{cc}
A_{0} X+X A_{0}^{\top}+b y+y^{\top} b^{\top}+\frac{2 e^{\mu}}{h} X+e^{\mu} A_{1} Z A_{1}^{\top} & X \\
X & \frac{-1}{e^{\mu}} Z
\end{array}\right) \leq 0,
$$

$\gamma X \geq X H+H X>0, X>0, Z H+H Z-\mu Z>0, Z>0$.

then the functional $Q$ defined by (5) with $P=X^{-1}$ and $S=$ $Z^{-1}$ satisfies the conditions $\left.C 1\right)$-C4) of Theorem 2 and the control defined by

$$
u(V, x(t))=V^{1-\mu} k D\left(V^{-1}\right) x(t),
$$

with $k=y X^{-1}, \quad V \in \mathbb{R}_{+}: Q\left(V, x_{h}(t)\right)=0$ stabilizes the origin of the system (4) hyper exponentially with degree $r=1$, the decay rate $\alpha=\frac{2 \mu}{\gamma h}$, where $\gamma=\max \left\{\lambda_{\max }\left(X^{1 / 2} H+H X^{1 / 2}\right)\right.$, $\left.\mu+\lambda_{\max }\left(Z^{1 / 2} H+H Z^{1 / 2}\right)\right\}$, and the domain of initial conditions $\Pi=\left\{\varphi \in \mathbb{C}_{h}: Q(1, \varphi)<0\right\}$. The control (7) is a continuous function of time and $\left\|u\left(V, x_{h}(t)\right)\right\| \leq V^{1-\mu} \sqrt{\lambda_{\max }\left(P^{-1 / 2} k^{T} k P^{-1 / 2}\right)}$ for $Q\left(V, x_{h}(t)\right)=0$.

Proof: I. The functional $Q(V, y(\cdot))$ defined by (5) is continuously F-differentiable in $\mathbb{R}_{+} \times \mathbb{C}_{h}$. Since $P>0, Q>0$ and $\mu \in(0,1)$ then $q_{i} \in \mathcal{I} \mathcal{K}_{\infty}, i=1,2$ and the inequalities

$\frac{\lambda_{\min }(P)\|y(0)\|^{2}+\lambda_{\min }(S) \int_{-h}^{0}\left(\frac{e}{V}\right)^{\frac{2 \tau+h}{h \mu^{-1}}}\|y(\tau)\|^{2} d \tau}{\max \left\{V^{2+2 \mu(n-1)}, V^{2}\right\}} \leq Q(V, y(\cdot))+1 \leq$ 


$$
\frac{\lambda_{\max }(P)\|y(0)\|^{2}+\lambda_{\max }(S) \int_{-h}^{0}\left(\frac{e}{V}\right)^{\mu \frac{2 \tau+h}{h}}\|y(\tau)\|^{2} d \tau}{\min \left\{V^{2+2 \mu(n-1)}, V^{2}\right\}}
$$

hold for all $V \in \mathbb{R}_{+}$and $y \in \mathbb{C}_{h}$. Hence, it is easy to see that for any $y \in \mathbb{C}_{h}$ there exists $V \in \mathbb{R}_{+}$such that $Q(V, y)=0$. Introduce the functions $q_{1}: \mathbb{R}_{+} \times \mathbb{R}_{+} \rightarrow \mathbb{R}$ and $q_{2}: \mathbb{R}_{+} \times$ $\mathbb{R}_{+} \rightarrow \mathbb{R}$ by the formulas $q_{1}\left(\sigma_{1}, s\right)=\frac{\lambda_{\min }(P) s^{2}}{\max \left\{\sigma_{1}^{2}, \sigma_{1}^{2+2 \mu(n-1)}\right\}}-$ 1 and $q_{2}\left(\sigma_{2}, s\right)=\frac{\left(\lambda_{\max }(P)+\lambda_{\max }(S) e^{\mu} \sigma_{2}^{\mu} h\right) s^{2}}{\min \left\{\sigma_{2}^{2}, \sigma_{2}^{2+2 \mu(n-1)}\right\}}-1$, where $\sigma_{1}, \sigma_{2}, s \in \mathbb{R}_{+}$. The obtained estimates also guarantee that $q_{1}(V,\|y(0)\|) \leq Q(V, y(\cdot)) \leq q_{2}\left(V,\|y\|_{h}\right)$ for all $V \in \mathbb{R}_{+}$ and $y \in \mathbb{C}_{h}$. The derivative of $Q(\cdot, y)$ for $y \in \mathbb{C}_{h}$ is

$$
\begin{gathered}
\frac{\partial Q(V, y)}{\partial V}=-\frac{y^{\top}(0) D\left(V^{-1}\right)(H P+P H) D\left(V^{-1}\right) y(0)}{V}- \\
\frac{\int_{-h}^{0}\left(\frac{e}{V}\right)^{\mu \frac{2 \tau+h}{h}} y^{\top}(\tau) D\left(V^{-1}\right)(H S+S H) D\left(V^{-1}\right) y(\tau) d \tau}{V}- \\
\frac{\mu \int_{-h}^{0} \frac{2 \tau+h}{h}\left(\frac{e}{V}\right)^{\mu \frac{2 \tau+h}{h}} y^{\top}(\tau) D\left(V^{-1}\right) S D\left(V^{-1}\right) y(\tau) d \tau}{V} .
\end{gathered}
$$

The inequalities (6) for $P:=X^{-1}$ and $S=Z^{-1}$ imply $\frac{\partial Q}{\partial V}<$ 0 for all $V \in \mathbb{R}_{+}$and $y \in \mathbb{C}_{h} \backslash\{0\}$. Therefore, the conditions C1)-C4) of Theorem 2 hold. Obviously, the condition $C 4^{*}$ ) of Theorem 5 also holds for $c=\sqrt{\lambda_{\max }(P)}$ and $V^{\max }=1$.

II. If $x(t)$ is a solution to the system (4), (7) then

$$
\begin{gathered}
\frac{\partial Q\left(V, x_{h}(t)\right)}{\partial t}= \\
2 x^{\top}(t) D\left(V^{-1}\right) P D\left(V^{-1}\right)\left(A_{0} x(t)+A_{1} x(t-h)+b u(t)\right)+ \\
e^{\mu} V^{-\mu} x^{\top}(t) D\left(V^{-1}\right) S D\left(V^{-1}\right) x(t)- \\
V^{\mu} e^{-\mu} x^{\top}(t-h) D\left(V^{-1}\right) S D\left(V^{-1}\right) x(t-h)- \\
\frac{2 \mu \ln \left(\frac{e}{V}\right)}{h} \int_{-h}^{0}\left(\frac{e}{V}\right)^{\frac{2 \tau+h}{h}} x^{\top}(t+\tau) D\left(V^{-1}\right) S D\left(V^{-1}\right) x(t+\tau) d \tau .
\end{gathered}
$$

Taking into account that $D\left(V^{-1}\right) A_{0} D^{-1}\left(V^{-1}\right)=V^{-1} A_{0}$, $D\left(V^{-1}\right) A_{1} D^{-1}\left(V^{-1}\right) \quad=\quad A_{1}, \quad D\left(V^{-1}\right) b u=$ $V^{-\mu} b k D\left(V^{-1}\right) x(t)$ we obtain

$$
\begin{gathered}
\frac{\partial Q\left(V, x_{h}(t)\right)}{\partial t}=-\frac{2 \mu \ln \left(\frac{e}{V}\right)}{h}+q^{\top} W q+ \\
\frac{2}{h}\left(\mu \ln \left(\frac{e}{V}\right)^{\mu}-\frac{e^{\mu}}{V^{\mu}}\right) x^{T}(t) D\left(V^{-1}\right) P D\left(V^{-1}\right) x(t),
\end{gathered}
$$

where

$$
\begin{gathered}
W=\left(\begin{array}{cc}
e^{-\mu}\left[P\left(A_{0}+b k\right)+\left(A_{0}+b k\right)^{\top} P\right]+S+\frac{2}{h} P & P A_{1} \\
A_{1}^{\top} P & -S
\end{array}\right), \\
q=\left(\begin{array}{c}
\sqrt{\frac{e^{\mu}}{V^{\mu}}} D\left(V^{-1}\right) x(t) \\
\sqrt{\frac{V^{\mu}}{e^{\mu}}} D\left(V^{-1}\right) x(t-h)
\end{array}\right) .
\end{gathered}
$$

Taking into account $\mu \ln \left(\frac{e}{V}\right) \leq \frac{e^{\mu}}{V^{\mu}}$ for $V \in \mathbb{R}_{+}$the LMIs (6) and Schur Complement imply $-\left[\frac{\partial Q}{\partial V}\right]^{-1} \geq$ $\frac{V}{\gamma}$ and $\frac{\partial Q\left(V, x_{h}(t)\right)}{\partial t} \leq-\frac{2 \mu \ln \left(e V^{-1}\right)}{h}$. Therefore, $\dot{V}=$ $-\left[\frac{\partial Q}{\partial V}\right]^{-1} \frac{\partial Q\left(V, x_{h}(t)\right)}{\partial t} \leq-\frac{2 \mu \ln \left(e V^{-1}\right) V}{\gamma h}$, for $V(y) \leq 1$. Theorem 5 implies that the system (4), (7) is hyper exponentially stable with the domain of hyper exponential attraction $\Pi$ (or equivalently for $V(\cdot) \leq 1$ ). The estimate of the hyper exponential convergence rate follows from $\sqrt{\lambda_{\min }(P)}\|y(0)\| \leq V$.

III. The control function $u$ is locally bounded. Indeed,

$$
\begin{aligned}
& \|u(V, x(t))\|^{2}=V^{2-2 \mu} x^{T}(t) D\left(V^{-1}\right) k^{T} k D\left(V^{-1}\right) x(t) \\
\leq & \lambda_{\max }\left(P^{-1 / 2} k^{T} k P^{-1 / 2}\right) V^{2-2 \mu} x^{T}(t) D\left(V^{-1}\right) P D\left(V^{-1}\right) x(t) .
\end{aligned}
$$

Hence, for $Q(V, y)=0$ we have $\|u(V, x(t))\|^{2} \leq$ $\lambda_{\max }\left(P^{-1 / 2} k^{T} k P^{-1 / 2}\right) V^{2-2 \mu}$. Since $V(\cdot)$ is a continuous functional then the function $u(\cdot):=u\left(V\left(x_{h}(\cdot)\right), x_{h}(\cdot)\right)$ is the continuous function and $u(t) \rightarrow 0$ as $V\left(x_{h}(t)\right) \rightarrow 0$.

The presented control scheme admits LMI constraints to parameters of nonlinear hyper exponential (fast) feedback. Any existing LMI solver can be used in order to adjust the control parameters. Note that introducing an additional LMI

$$
\left(\begin{array}{ll}
X & y^{T} \\
y & u_{0}^{2}
\end{array}\right) \geq 0, \quad u_{0} \in \mathbb{R}_{+}
$$

to (6) we guarantees $\|u(t)\| \leq u_{0}$ for $V \leq 1$. Indeed, the inequality $\lambda_{\max }\left(P^{-1 / 2} k^{T} k P^{-1 / 2}\right) \leq u_{0}^{2}$ can be equivalently rewritten as $y^{T} y \leq u_{0}^{2} X$ and Schur Complement implies (8).

\section{Practical implementation}

In order to apply the control (7) in practice it is necessary to present the procedure for calculation of the value $V$ for any given $y(\cdot) \in \mathbb{C}_{h}$ such that $Q(V, y(\cdot))=0$. Since $Q$ satisfies the condition $C 3$ ) of Theorem 2 then for any $y(\cdot)$ the ILF equation has a unique solution, which can be easily found by means of, for example, the bisection method.

An alternative approach is to realize the ILKF-based control scheme using a Newton method for numerical calculation of the solution of the equation $Q(V, y(\cdot))=0$, for example,

$$
\dot{V}_{n}=-p Q\left(V_{n}, y(\cdot)\right)\left[\frac{\partial Q\left(V_{n}, y(\cdot)\right)}{\partial V_{n}}\right]^{-1}, \quad p \in \mathbb{R}_{+} .
$$

For any fixed $y(\cdot)$ the presented gradient method guarantees $V_{n} \rightarrow V: Q(V, y(\cdot))=0$. In order to boost the convergence of Newton method its fast modification can be considered (see, for example, [5]). In practice, the ILKF control can be realized in a sufficiently fast digital control device.

\section{Numerical Simulation}

Consider the problem of hyper exponential stabilization of the system (4) with $n=2$, the matrix $A_{1}=\operatorname{diag}\{1,0\}, \mu=$ $0.75, u_{0}=50$ and the delay $h=0.5$. By solving the LMI system (6) we obtain $K=\left(\begin{array}{ll}-82.3072 & -14.6158\end{array}\right)$ and

$$
P=\left(\begin{array}{ll}
6.3785 & 0.7197 \\
0.7197 & 0.1081
\end{array}\right), \quad S=\left(\begin{array}{ll}
1.5866 & 0.0032 \\
0.0032 & 0.0008
\end{array}\right)
$$

The simulations for the ILKF control application have been made in MATLAB Simulink. The value of ILKF was calculated on-line using the Newton method (9) with $p=$ $10^{3}$. All integrals, which appear in (9), were approximated by Simpson formula. In order to avoid singularities during calculation of $Q(V, y(\cdot))$ the admissible value of $V$ is also bounded from below by $V_{\min }=0.01$. In this case the hyper exponential convergence can only be guaranteed to a zone defined by inequality $V(y) \leq V_{\min }$. However, such assumption is not restrictive from practical point of view, since due to measurement noises and uncertainties just a practical stability is possible in real-life systems. The results of simulations are depicted in Figure 1. 


\section{CONCLUSIONS}

This technical note presents the method of the implicit Lyapunov-Krasovski functionals and demonstrates its possible application to control design. The obtained stability theorems extend the results of the paper [23] to time-delay systems. The main difficulty of this extension is the representation of all conditions of Lyapunov-Krasovski theorems using the implicit functions in Banach spaces (see, conditions C1)-C5) of Theorem 2). The special class of implicit functions $\mathcal{I} \mathcal{K}_{\infty}$ is introduced for this purpose.

The application ILKF method for fast (hyper exponential) stabilization of a time-delay system is demonstrated on an example. The corresponding ILKF has been obtained by means of an appropriate parametrization of the classical explicit Lyapunov-Krasovski functional. The hyper exponential stabilization of a more general time-delay model is a possible direction of future research.

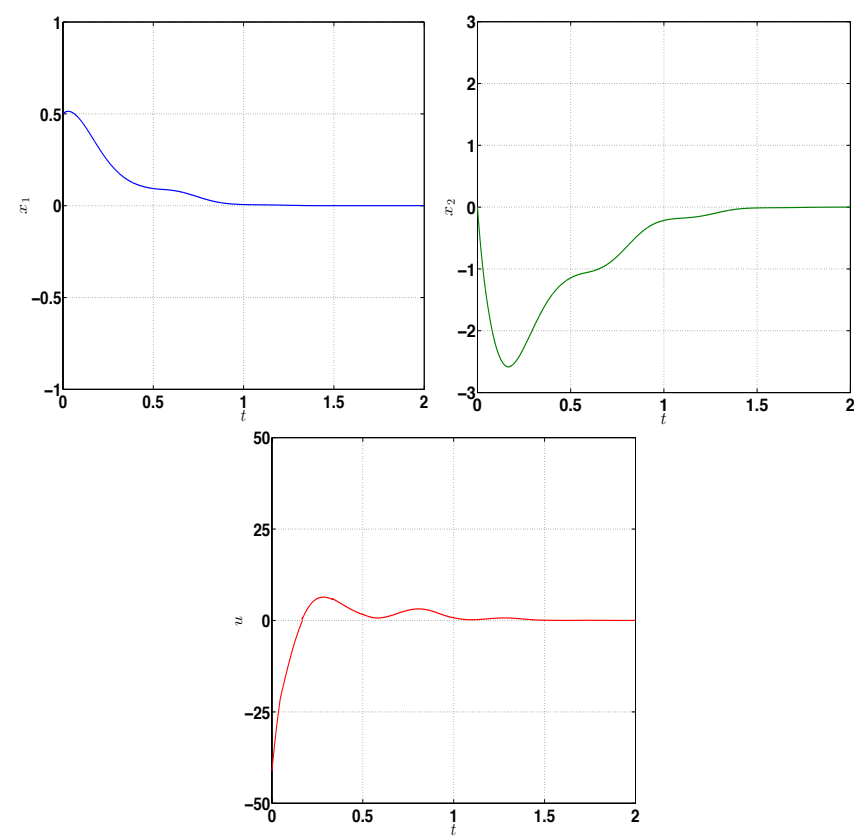

Fig. 1. The simulation results ( $x_{1}$ - blue line; $x_{2}$ - green line; $u$ - red line).

\section{REFERENCES}

[1] J. Adamy and A. Flemming. Soft variable-structure controls: a survey. Automatica, 40:1821-1844, 2004.

[2] A. Bacciotti and L. Rosier. Lyapunov Functions and Stability in Control Theory. Springer, 2nd edition, 2005.

[3] S.P. Bhat and D.S. Bernstein. Finite-time stability of continuous autonomous systems. SIAM Journal of Control and Optimization, 38(3):751-766, 2000.

[4] B. Biemond, N. van de Wouw, and H. Nijmeijer. Bifurcations of equilibrium sets in mechanical systems with dry friction. Physica D, 241:1882-1894, 2012.

[5] J. Cortes. Finite-time convergent gradient flows with applications to network consensus. Automatica, 42(11):1993-2000, 2006.

[6] R. Courant and F. John. Introduction to calculus and analysis (Vol. II/1). New York: Springer, 2000.
[7] B.K. Driver. Analysis Tools with Applications. Springer, 2003.

[8] D. Efimov, A. Polyakov, E. Fridman, W. Perruquetti, and J.-P. Richard. Comments on finite-time stability of time-delay systems. Automatica, (DOI: 10.1016/j.automatica.2014.05.010), 2014.

[9] E. Fridman. Descriptor discretized lyapunov functional method: Analysis and design. IEEE Transactions on Automatic Control, 51(5):890-897, 2006.

[10] K. Gu, V. Kharitonov, and J. Chen. Stability of TimeDelay Systems. Boston, MA: Birkhauser, 2003.

[11] V.T. Haimo. Finite time controllers. SIAM Journal of Control and Optimization, 24(4):760-770, 1986.

[12] J. Hale. Theory of Functional Differential Equations. Springer-Verlag, 1977.

[13] Y. Hong. Finite-time stabilization and stabilizability of a class of controllable systems. Systems \& Control Letters, 46(4):231-236, 2002.

[14] K. Jittorntrum. An implicit function theorem. Journal of Optimization Theory and Applications, 25(4):575-577, 1978.

[15] V.L. Kharitonov. Robust stability analysis of time delay systems: A survey. Annual Review in Control, 23:185196, 1999.

[16] V.I. Korobov. Solution of a synthesis problem using a controllability function. Doklady Academii Nauk SSSR, 248:1051-1055, 1979.

[17] M. Krstic. Delay Compensation for Nonlinear, Adaptive, and PDE Systems. Birkhauser Boston, 2009.

[18] J. Macdonnell. Some critical points on the hyperpower function ${ }^{n} x=x^{x^{x^{\prime}}}$. International Journal of Mathematical Eduction, 20(2):297-305, 1989.

[19] E. Moulay, M. Dambrine, N. Yeganefar, and W. Perruquetti. Finite-time stability and stabilization of time-delay systems. System \& Control Letters, 57(7):561-566, 2008.

[20] E. Moulay and W. Perruquetti. Finite-time stability and stabilization: State of the art. Lecture Notes in Control and Information Sciences, 334:23-41, 2006.

[21] Y. Orlov. Discontinous systems: Lyapunov analysis and robust synthesis under uncertainty conditions. SpringerVerlag, 2009.

[22] A. Polyakov. Nonlinear feedback design for fixed-time stabilization of linear control systems. IEEE Transactions on Automatic Control, 57(8):2106-2110, 2012.

[23] A. Polyakov, D. Efimov, and W. Perruquetti. Finite-time stabilization using implicit lyapunov function technique. In 9th Symposium on Nonlinear Control Systems, , pages 140-145, 2013.

[24] A. Polyakov, D. Efimov, W. Perruquetti, and J.-P. Richard. Implicit Lyapunov-Krasovski Functionals for Time Delay Systems. In 53rd Conference on Decision and Control, 2014.

[25] J.-P. Richard. Time-delay systems: An overview of some recent advances and open problems. Automatica, 39(10):1667-1694, 2003.

[26] E. Roxin. On finite stability in control systems. Rendiconti del Circolo Matematico di Palermo, 15(3):273-283, 1966. 